
internationales

vol. 32 - $n^{\circ} 1 \mid 2016$

Les migrations des Roms roumains en Europe

\title{
Migrations nigérianes à l'écran : Man on Ground et l'Afrique du Sud post-apartheid
}

Nigerian Migration on Screen: Man on Ground and Post-Apartheid South Africa

Migraciones nigerianas en pantalla: Man on Ground y Sudáfrica postapartheid

\section{Françoise Ugochukwu}

\section{(2) OpenEdition}

\section{Journals}

Édition électronique

URL : https://journals.openedition.org/remi/7605

DOI : $10.4000 /$ remi. 7605

ISSN : $1777-5418$

Éditeur

Université de Poitiers

Édition imprimée

Date de publication : 1 mars 2016

Pagination : 211-228

ISBN : 979-10-90426-27-6

ISSN : 0765-0752

Référence électronique

Françoise Ugochukwu, « Migrations nigérianes à l'écran : Man on Ground et l'Afrique du Sud postapartheid », Revue européenne des migrations internationales [En ligne], vol. 32 - n¹ | 2016, mis en ligne le 01 mars 2018, consulté le 14 avril 2022. URL : http://journals.openedition.org/remi/7605 ; DOI : https://doi.org/10.4000/remi.7605 


\section{Migrations nigérianes à l'écran : Man on Ground et l'Afrique du Sud post-apartheid}

\section{Françoise Ugochukwu'}

Si le Nigeria a toujours connu une importante émigration, la récession qui a frappé le pays dans les années 1980 et les restrictions imposées à l'immigration par les pays occidentaux ont poussé de nombreux Nigérians, qui avaient jusque-là émigré plutôt vers I'Europe et les États-Unis pour des raisons historiques, à explorer le reste du continent africain, plus particulièrement l'Afrique australe et I'hémisphère sud, à la recherche d'un nouvel Eldorado. Statistiques et études confirment depuis quelques années une diversification des pays ciblés par les migrations africaines, avec moins de $5 \%$ des migrants cherchant désormais à atteindre le Nord et leur préférant les pays voisins de chez eux, I'Afrique australe ou ce nouveau pays de cocagne, I'Afrique du Sud. Nséké (2012) note ainsi que " si la Côte d'Ivoire exerce un fort potentiel d'attraction, I'Afrique du Sud demeure le pays qui accueille le plus d'immigrés, réfugiés compris. Victime de sa réussite économique et surtout de son émergence fulgurante, la nation "arc-en-ciel" dispose d'une population immigrée qui compte pour 3 à $5 \%$ de la population totale du pays. [...] Comme dans les autres points du globe, on remarque donc que le principal facteur de mobilisation des migrants reste la recherche de meilleures conditions de vie".

\section{La migration au cinéma}

La réflexion sur la migration est depuis longtemps I'un des grands thèmes de la littérature africaine. Qu'en est-il du cinéma ? Si un grand nombre de films sur les itinéraires et l'expérience des migrants d'autres continents ont déjà été produits dans les pays occidentaux, comme l'attestent plusieurs ouvrages récents (Deveny, 2012 ; Schrader et Winkler, 2013 ; Rofheart, 2014), rares sont pour l'instant les films africains qui ont placé ce sujet au centre de leurs scénarios. Le documentariste français Boris Lojkine partageait récemment son exploration de la migration dans le blog du Nouvel Observateur, "Rues d'Afrique ", qui " veut mettre en valeur des regards originaux et décalés sur l'actualité des Afriques, continent pluriel ". II y expliquait l'origine de Hope (2014), son documentaire sur les migrants africains : "Depuis très longtemps, je lisais des articles dans la

1 Development Policy \& Practice, The Open University, Walton Hall, Milton Keynes MK7 6AA, UK ; francoise.ugochukwu@open.ac.uk 
presse, des récits de voyage de migrants, et je trouvais que c'était exactement le genre d'histoires faites pour le cinéma que j'ai envie de faire " (Cessou, 2015).

Au Nigeria, si les ouvrages de littérature restent peu lus en dehors de l'école, notamment en raison de leur coût, il convient de rappeler que l'avènement du nouveau cinéma nigérian, plus connu sous le nom de Nollywood, a permis au grand public, depuis 1992, de visionner ces films produits dans le sud du pays et vendus sur tous les marchés ${ }^{2}$. Jedlowski rappelle que " cette industrie, apparue au début des années 1990, est aujourd'hui considérée comme l'une des plus vastes au monde. Les vidéos films qu'elle produit, le plus souvent des productions à petits budgets sorties directement sur vidéo et disséminées par l'intermédiaire de réseaux transnationaux informels, sont aujourd'hui disponibles dans toute l'Afrique et dans la plupart des pays où vivent des personnes d'origine africaine " (Jedlowski, $2015: 397)^{3}$. Le succès du cinéma nigérian ne s'est, depuis, jamais démenti, renforcé par son côté didactique. Producteurs et réalisateurs s'appuient sur le formidable pouvoir de l'image pour faire passer leur message : réflexion sur les maux du pays, dénonciation de pratiques variées et offre de conseils par scénarios interposés.

Ces films, inspirés par la vie quotidienne et les faits divers, sont perçus par le public populaire comme une image à peine retouchée de la réalité, ce qui a largement contribué à l'explosion de la production depuis 1999, date du retour aux gouvernements civils au Nigeria. Estimée à un minimum de 7000 films il y a déjà dix ans (Barrot, 2005 : 5), cette production s'exporte depuis dans toute l'Afrique, à commencer par les pays anglophones (Hungbo, 2014 : 174). Beaucoup de choses ont changé, par ailleurs, depuis le début des années 1990, et les savoir-faire de cette industrie se sont nettement améliorés, permettant un usage accru des gros plans et de la voix off et une meilleure qualité du son et du travail post-filmique. Le contenu des films et la façon d'aborder les sujets traités ont également évolué, avec, en particulier, une plus grande place donnée aux trajectoires individuelles et aux espaces diasporiques, en même temps que se multipliaient les coproductions internationales.

Décrite comme " edutainment " - éducation + divertissement (education + entertainment) -, cette production reste avant tout porteuse d'un message, adressé, non pas seulement aux individus, mais aussi aux groupes. Visionnés en famille à la maison, ou dans des lieux publics tels que cafés ou salles (Ugochukwu, 2013 : 181), ces films alimentent ensuite les conversations et sont même repris dans les enseignements des Églises.

Nollywood a, dès ses débuts et pour des raisons historiques, intégré une réflexion sur la migration, phénomène qui touche tout le sud du Nigeria depuis l'époque coloniale (Haynes, 2013 : 74). Les premiers films de Nollywood situés partiellement ou totalement à l'étranger ont suivi et mis à l'écran l'évolution de la migration nigériane, s'appuyant sur l'illusion que I'Europe et l'Amérique étaient les mieux placées pour assurer le bonheur et la prospérité des Nigérians (Ugochukwu, 2012). Les scénarios sont donc, depuis le début, fortement marqués

2 Pour plus de détails sur Nollywood, voir Ugochukwu (2013) qui offre en outre une bibliographie exhaustive sur le nouveau cinéma nigérian.

3 Toutes les citations en anglais ont été traduites par l'auteur de cet article. 
par l'importance que revêt la migration pour les jeunes générations (Jedlowski, 2012 : 239), comme l'illustrent les quinze films étudiés ici, produits entre 2002 et 2015 et brièvement considérés de façon à la fois chronologique et thématique à titre de comparaison. Haynes, notant le nombre grandissant des metteurs en scène et des réalisateurs nigérians en diaspora et des films traitant de la migration, a récemment défini ces films tournés à l'étranger comme un genre à part entière (Krings et Okome, 2013b : 71), " dans un contexte globalisé au sein duquel les personnes et les médias se déplacent ensemble " (Pype, 2013 : 201). Ces nouveaux films sont soutenus, non seulement par la vaste diaspora nigériane, mais aussi par les déplacements et le profil international des acteurs, actrices et réalisateurs nollywoodiens (Tsika, 2015).

Si l'étude de ce nouveau cinéma populaire occupe de plus en plus de jeunes chercheurs, le traitement de la migration et de son impact dans les films nigérians n'a cependant pas été vraiment considéré jusqu'ici. Cet article, rédigé dans un contexte de malaise diplomatique entre le Nigeria et l'Afrique du Sud largement répercuté dans les médias, considère d'abord l'évocation de la figure de l'étranger dans la production nollywoodienne et l'évolution de son traitement de la migration depuis les débuts. II brosse ensuite un tableau des difficultés rencontrées par les migrants africains en Afrique du Sud, pour mieux contextualiser le film Man on Ground (2011) du Sud-Africain d'origine nigériane Akin Omotoso, dont l'analyse est l'objet de l'article. L'étude du film s'intéresse à son traitement de la migration nigériane et africaine en Afrique du Sud, à son interprétation du malaise noté précédemment et au commentaire audiovisuel qui y est fait de la communication interculturelle ou de son absence. La dernière partie de l'article rend compte de l'utilisation politique et pédagogique faite de ce film et de son impact, dans la lignée de celle des films nollywoodiens traditionnels. Les sources se composent d'ouvrages publiés pour la partie historique, de médias en ligne (presse et blogs) pour l'information qu'ils apportent sur les relations entre Nigeria et Afrique du Sud, et de quinze autres films nigérians pour la lecture qui y est faite de la migration nigériane.

\section{L'évolution du regard sur la migration}

Le 23 septembre 2012, un Nigérian écrivait dans son blog, sous le titre Le voyage des immigrants africains : " chaque fois que je rencontre des immigrants africains, je me demande quelle est leur histoire - leur voyage vers une vie meilleure à l'étranger. Chaque année, des centaines de milliers d'Africains quittent leur pays d'origine [...] parce qu'ils croient qu'une vie meilleure les attend à l'étranger ${ }^{4}$. Les vidéos films nigérians semblent les mieux placés pour répondre à cette question puisqu'ils sont devenus en quelque sorte les carnets de bord des migrants. Ils les suivent d'abord du village à la ville - Lagos ou Abuja -, avant de les accompagner à l'aéroport et vers l'inconnu qui, dans l'imagerie populaire nigériane, a longtemps été un lieu paradisiaque, mais chargé de mystère. Une première catégorie de films, datant des années 2000 , reproduit la situation décrite dans Le Malaise d'Achebe (1960), mettant en scène un jeune Nigérian de retour de l'étranger, à l'image des enfants de cette classe moyenne envoyés en Europe ou en Amérique dans les années 1960 et 1970 pour y poursuivre leurs études.

4 Cf. http://omonaij.wordpress.com/2012/09/23/the-journey-of-african-immigrants 
"Les migrations des Nigérians s'articulent dans un espace migratoire dont les deux composantes, l'espace de départ et l'espace d'accueil, sont chargées d'une dimension historique importante "(Weiss, $1998: 75)$. Ces films se sont d'abord contentés d'évoquer cette histoire au passé, commentée dans les conversations ou visualisée par le biais d'un mobilier importé, d'écrans de télévision montrant des films étrangers, ou des tenues vestimentaires de ces jeunes filles de retour d'Europe en tee-shirts et jeans étroits. Plus tard, les films nigérians ont mis en scène le retour des héros - un soldat par exemple, rentré après avoir terminé sa formation militaire en Angleterre, promu au rang de général et propriétaire d'un pavillon Iondonien (Fateful Love, 2004); ou d'autres, auréolés de gloire après l'obtention de diplômes universitaires (Widow, 2007 ; The King's Secret, 2012). Dans tous ces films, la migration est présentée comme la solution à tous les problèmes domestiques - dans Widow (2007) par exemple, une histoire vraie qui se clôt sur la fuite de la veuve maltraitée et de ses enfants vers l'aéroport et la Grande-Bretagne.

Ce n'est là que le début d'une longue exploration qui va progressivement permettre aux spectateurs nigérians de découvrir la face cachée de l'étranger ${ }^{5}$. La première scène d'Osuofia in London (2003) illustre parfaitement les clichés prétendument naïfs sur " obodo oyibo" ("le pays des Blancs "). On y apprend que le frère du villageois Osuofia, parti depuis si longtemps qu'on I'avait oublié, vient de mourir en Angleterre en léguant une importante somme d'argent à son frère. La première scène de Fateful Love (2004) promène le spectateur dans le secteur touristique de Londres, de Westminster à "Big Ben " et au palais de Buckingham. Nollywood rassemble ainsi peu à peu un kaléidoscope d'images évoquant l'étranger, du millionnaire au téléphone, mentionnant ses conteneurs en transit, à la fille du chef établie en Europe et y vivant à l'aise. Ces films mettent l'accent sur les gains des migrants : I'éducation, l'émancipation loin des pressions familiales et la prospérité. Le personnage principal du film comique Brainwash (2006) donne un autre visage à ces clichés : escroc à la petite semaine de retour de Côte d'Ivoire où il s'est fait passer pour un grand commerçant, il sort de l'aéroport, un lourd manteau sous le bras, après avoir mis des boucles d'oreilles (marqueurs du féminin ${ }^{6}$ au Nigeria et donc lues comme l'une de ces modes décadentes venues d'Occident), pour rehausser son profil en laissant croire qu'il revient d'Allemagne.

Une autre catégorie de films raconte I'histoire de nouveaux migrants doctorants, spécialistes de l'import-export ou cousins en partance pour rejoindre un membre de leur famille établi ailleurs. Les Nigérians n'ont longtemps connu de l'étranger que les mandats reçus de leurs membres expatriés et la vaste maison construite en un rien de temps - une image dorée jalousement protégée par tous ceux de retour d'Occident -, dont aucun d'entre eux ne pouvait, sans

5 Le Nigeria, longtemps victime de son instabilité politique et de conflits internes, s'est trouvé du même coup coupé de l'étranger, du fait de la faiblesse de ses communications (poste et télécommunications entre autres).

6 On met des boucles d'oreille aux fillettes dès la naissance, ce qui évite aux visiteurs de demander le sexe du bébé. 
risquer d'être incompris, avouer les difficultés et les souffrances de l'exil ${ }^{7}$. La poursuite de l'exploration de l'Occident par Nollywood finit par écorner cette image idyllique, à travers des films comme One Dollar (2002), sombre comédie qui brise le rêve américain sous les yeux de villageois nourris de stéréotypes et aussi ignorants qu'ambitieux. Les parents d'une jeune fille dont le prétendant, un instituteur, paie les études et soutient la famille, reçoivent la visite d'un riche voisin venu leur demander la main de leur fille pour son fils établi aux États-Unis. Éblouis par la perspective d'un beau-fils " américain " et attirés par la liasse de dollars offerts par I'homme d'affaires, les parents de la jeune fille rejettent le prétendant en faveur du migrant inconnu, qui se révélera être un dangereux drogué.

D'autres films ont contribué à cette éducation populaire en renversant la vue manichéenne traditionnelle opposant les mérites de l'Occident aux inconvénients de la vie au pays. C'est le cas, entre autres, d'African Dilemma (2006) qui met en avant la réponse du principal protagoniste concernant le besoin d'acquérir la fameuse carte verte, considérée au Nigeria comme équivalant à un sauf-conduit susceptible de faciliter l'immigration légale en direction des ÉtatsUnis. Deux autres, Life in New York et Mr. Ibu in London, tous les deux sortis en 2004 et partiellement tournés en Occident, attirent I'attention des Nigérians sur l'envers du " pays des Blancs ". Life in New York (2004) par exemple, bâti autour d'une histoire d'adultère, révèle les difficultés des immigrants confrontés à un univers athée où les traditions et les croyances africaines n'ont plus leur place. À la fin du film Mr. Ibu in London (2004), le personnage principal se retrouve à Lagos dans une situation pire que celle qu'il a quittée. Les films illustrant les tentations de I'Occident se focalisent sur trois thèmes : la drogue, la fraude et la prostitution. Un autre film, tourné partiellement en Amérique, lje the Journey (2010), met, de plus, en évidence la violence qui affecte la vie quotidienne dans certains espaces aux États-Unis. Weiss (1998: 41) souligne que « les vicissitudes auxquelles est soumis I'espace migratoire révèlent la grande adaptabilité des migrants nigérians. Leur esprit de self-made man et leur volonté farouche de réussir leur migration sont caractéristiques ". Ces films révèlent cependant souvent des Nigérians naïfs, isolés, jetés dans des situations qu'ils ne maîtrisent pas, cibles faciles et victimes toutes désignées, et confirment que " ces voyages de migrants qui auraient dû s'apparenter à des aventures ou à des expériences parfois dures, certes, mais riches en enseignement, ne semblent pas s'améliorer avec le temps, au contraire " (Crouillère, $2010: 176)$.

\section{De Lagos à Johannesburg}

Si de nombreux expatriés nigérians reviennent aujourd'hui au pays, attirés par la promesse de meilleures conditions de vie (Fisher, 2011), I'étranger, même $s^{\prime}$ il est en grande partie démystifié, continue de faire rêver. Après des décennies

7 L'une de ces histoires a été publiée en ligne le 5 avril 2012 (cf. http://www.naijaurban. com/forty-years-nigerian-woman-enugu-victims-mass-shooting-oakland-california/ - le site originel n'est plus disponible), celle de "Mme Doris Chibuko, [...] identifiée comme I'une des victimes du tueur solitaire qui ouvrit le feu sur des étudiants et enseignants de l'université d'Oikos, dans I'est d'Oakland, en Californie [...]. Doris Chibuko, quarante ans, faisait des études d'infirmière à I'université et a été tuée juste deux mois avant son diplôme. [...] Native de l'État d'Enugu, elle avait exercé le droit au Nigeria avant d'émigrer aux États-Unis en 2002 avec son mari Efanye, après leur mariage cette même année ". 
de migrations vers l'Europe, " rançon de leur succès économique, certains pays africains sont [ainsi] devenus, au cours des dernières décennies, un véritable pôle d'immigration à l'échelle du continent "(Mboungou, 2012) et parmi eux, I'Afrique du Sud. C'est aussi celui qui compte le plus grand nombre de migrants africains - soit environ 2,4 millions - souvent victimes de xénophobie, de discrimination et de violences.

Comme le montre le film du réalisateur sud-africain d'origine nigériane Akin Omotoso, Man on Ground (Un homme à terre) (2011), "le chemin qui mène là-bas est long et difficile, et ceux qui parviennent à destination ne sont pas forcément mieux lotis " (Pitron, 2012a). C'est qu' en Afrique du Sud, la présence des immigrés et l'incertitude quant à leur nombre nourrissent une solide xénophobie. Le pourcentage de cette population étrangère serait de $3 \%$ à $5 \%$ par rapport à la population totale [...], ce qui accentue davantage le sentiment antiimmigrant dans la "nation arc-en-ciel" et les incidents violents à l'encontre des étrangers à qui, comme ailleurs, on attribue tous les maux, de la délinquance au chômage et même au sida. Les Africains francophones, les Mozambicains, les Malawites et les Zimbabwéens sont donc devenus une cible toute désignée. Les Sud-Africains noirs, longtemps confinés dans les townships et les bantoustans, les ont pris en grippe, car ils les suspectent de voler leur travail et de profiter indûment des investissements ou des services qu'ils ont si longtemps attendus " (Mboungou, 2012 ; Tati, 2008).

Les relations entre le Nigeria et l'Afrique du Sud, sont quant à elles restées " glaciales " de I'indépendance du Nigeria en 1960 jusqu'en 1994, du fait de I'hostilité de ce dernier au régime de l'apartheid (Ibeanu et Orji, 2004). De 1994 à 1998, le Nigeria, qui soutenait les Sud-Africains noirs, a ensuite bénéficié d'excellentes relations avec la population, situation qui a encouragé de nombreux Nigérians à émigrer. Les relations entre les deux pays se sont ensuite rapidement détériorées, les Sud-Africains accusant les Nigérians de trafic de drogue et d'autres maux.

L'Afrique du Sud, nation " la plus moderne, la plus diversifiée et la plus performante du continent " (Véron, 2006 : 168), est aujourd'hui devenue un partenaire incontournable du Nigeria sur le plan économique. Le commerce entre les deux pays, considérés comme " les deux plus puissants pays d'Afrique sub-saharienne " (Véron, 2006 : 164), a été multiplié par cinq depuis 2002 pour atteindre 3 milliards d'euros environ en 2012 (20 minutes, 2013) ce qui n'a pas pour autant réduit les tensions entre ces deux pays, celles-ci affectant en particulier les migrants nigérians qui sont plus de 250000 dont plus de 400 sont en prison ${ }^{8}$. L'écrivaine nigériane Chimamanda Adichie avait dès 2009 attiré l'attention sur le malaise entre les deux communautés et posé la question :

8 Cf. http://www.seneweb.com/news/Afrique/expulsion-de-nigerians-l-rsquo-afriquedu-sud-presente-ses-excuses-au-nigeria_n_61224.html ; http://french.people.com. cn/96852/7750153.html ; http://premiumtimesng.com/news/133063-jonathan-shocked-byhuge-number-of-nigerians-in-south-african-prisons.html 
"pourquoi les Sud-Africains détestent-ils les Nigérians ? " ${ }^{9}$. Ces derniers, poursuivis par la mauvaise réputation de leur pays, accusé d'être le champion de la contrefaçon, se plaignent en particulier des tracasseries administratives récurrentes et ressenties comme xénophobes dont ils sont victimes lors de leurs déplacements en Afrique du Sud. Par ailleurs, dans un entretien donné le 11 août 2013 à Johannesburg à I'occasion de I'inauguration de l'association des Nigérianes unies en Afrique du Sud, I'UNWISA, sa présidente de l'époque, Lindelwa Uche, menaçait d'organiser une manifestation afin de protester contre la discrimination dont sont quotidiennement victimes, dans leur propre pays, les Sud-Africaines mariées à des Nigérians ${ }^{10}$.

Ces discriminations répétées ont conduit en 2013 le ministre nigérian des Affaires étrangères, Olugbenga Ashiru, à déplorer officiellement " les attitudes négatives du gouvernement sud-africain contre des ressortissants nigérians en Afrique du Sud ". Selon lui, " le problème de fond est lié à l'attitude du citoyen sud-africain ordinaire envers les Nigérians. Ils ont une attitude xénophobe envers les Nigérians. Nous devons y remédier au sein de la commission bilatérale entre les deux pays. D'autres questions à discuter vont porter sur la procédure d'immigration, l'expulsion des Nigérians, le harcèlement de la police et I'attitude envers les Nigérians ". La présidente du Comité sur la diaspora de la Chambre nigériane des représentants, Abike Dabiri-Erewa, a elle-même rendu visite la même année au consulat sud-africain à Abuja, à la tête d'une délégation chargée d'exprimer l'inquiétude de son gouvernement concernant le nombre grandissant de Nigérians tués en Afrique du Sud. La présidente de la commission des affaires étrangères de la Chambre des représentants, Nnenna ElenduUkeje, a de son côté exprimé la préoccupation de sa commission, suite à la série d'attentats perpétrés contre les Nigérians et ses ambassades ${ }^{11}$. La question soulevée par Adichie a été reprise en ligne le 20 juin 2013 en ces termes : "Après la pauvreté de la population noire d'Afrique du Sud, I'intolérance est devenue I'un des legs durables de l'ère de l'apartheid. [...] Avant 1994, si les immigrants venus du reste du continent étaient en butte à la discrimination et même à la violence, la majorité de ces risques provenaient du racisme institutionnel de l'époque. Après 1994 et suite à la démocratisation, les cas de xénophobie se multiplièrent de façon surprenante. Entre 2007 et 2008, plus de soixante-sept personnes périrent du fait de crimes motivés par la haine. En 2008, une série d'émeutes laissèrent soixante-deux morts au cours d'une attaque apparemment motivée par la xénophobie. II convient de noter, cependant, que les immigrants africains ont été victimes d'attaques racistes, et que les Nigérians sont particulièrement visés par la violence haineuse et les incendies " (Naij.com, 2013).

9 The Guardian, 5 octobre 2009 (cf. http://www.I3.ulg.ac.be/adichie/cnaprimlinks. html\#interviews). Il faut cependant noter que d'autres communautés africaines ont elles aussi usé de violence vis-à-vis des Nigérians. Selon Weiss (1998: 69-70), " au fil des ans, la minorité nigériane du Cameroun indépendant a constamment été exposée à la discrimination et à l'intimidation [...] de la part d'une population autochtone qui a continué à envier leur succès économique. [...] Les rafles, les saisies illégales, les rackets musclés, les sévices corporels, les violences physiques, les séquestrations et les humiliations dont sont victimes les Nigérians pourraient nourrir les rapports des organisations humanitaires et des droits de l'homme ".

10 Cf. http://www.vanguardngr.com/2013/08/s-african-women-married-to-nigeriansthreaten-protest/\#sthash. SxVPPfQ3.dpuf

11 Cf. http://guineeinformation.fr/index.php/cedeao/141-green-tech1812688467/1690-incident-diplomatique-nigeria-afrique-du-sud (ce site n'est plus disponible), 7 mars 2012. 
Depuis, la situation a encore empiré. Selon les médias, le consul du Nigeria en Afrique du Sud, Ajulu Okeke, rapportait le 19 avril 2015 que ses compatriotes avaient perdu plus de 21 millions de rands du fait des récentes attaques xénophobes (BellaNaija.com, 2015 ; Premium Times, 2015), et Reuters rapportait le 28 avril de la même année le rappel temporaire de l'ambassadeur du Nigeria en Afrique du Sud après une nouvelle série d'émeutes xénophobes à Johannesburg (Brock et Dludla, 2015).

\section{De la vie à l'écran}

C'est dans ce climat de tensions que s'inscrit le second film du Sud-Africain d'origine nigériane Akin Omotoso, dont le premier film, God is African (2003), inspiré par les mouvements migratoires intra-africains, " thème d'ailleurs rarement traité " (Crouillère, 2010 : 190), avait " marqué un tournant dans I'histoire des réalisations d'immigrants africains en Afrique du Sud " (Dovey, 2009 : 144). Ce premier film, dont I'action se déroulait dans I'Afrique du Sud de 1995, mettait en scène un étudiant nigérian, Femi, cherchant à sensibiliser son université à la question des droits de l'homme par le biais de la radio locale au sujet du poète nigérian Saro-Wiwa ${ }^{12}$. Une poignée de films nigérians appartenant au Nollywood traditionnel (Coming to South Africa, 2005 ; Akpegi Boyz, 2009) ont également cherché à attirer l'attention sur les dérives de la migration, dans la lignée directe de productions similaires. D'autres films sud-africains ont depuis " souligné l'inquiétude des metteurs en scène concernant une réalité sociale caractérisée par la violence, et offert différentes façons de conceptualiser, de visualiser et de critiquer cette violence " (Dovey, $2009: 6$ ).

Man on Ground (2011) raconte I'histoire de deux demi-frères, Ade et Femi, qui ont quitté le pays yoruba du Nigeria. Ade est l'exemple d'une immigration réussie : il est banquier à Londres ; Femi, lui, dissident politique au pays, a choisi de se réfugier en Afrique du Sud où il vit de petits métiers dans un ghetto noir de Johannesburg. Profitant d'une courte visite dans la ville, Ade cherche à joindre son frère pour lui donner un paquet de la part de sa mère et découvre qu'il a disparu depuis une semaine. II se met alors à sa recherche, s'appuyant sur les bribes d'information recueillies, et apprend peu à peu ce qui constituait le quotidien de Femi et ses difficultés. Sa quête le conduit chez le patron de Femi, Timothi, qui vit dans I'un des ghettos de la ville, mais, ni Timothi, ni sa femme Lindiwe, ne se montrent prêts à répondre aux questions d'Ade. Pendant que ce dernier se trouve avec Timothi, une émeute à caractère xénophobe éclate parmi les Sud-Africains noirs et les deux hommes se trouvent forcés de passer la nuit ensemble dans le bureau deTimothi. Les longues heures passées à boire en silence, I'un en face de l'autre, seront pour Ade I'occasion de finalement comprendre comment Femi a tenté de survivre en Afrique du Sud et ce qui lui est arrivé.

Le film fait partie de ce que Haynes définit comme "la nouvelle vague " du cinéma nigérian, " un mouvement qui se démarque des productions nollywoodiennes habituelles "(Jedlowski, $2013: 37)$. Ces films, qualifiés par certains

12 Ken Saro-Wiwa (10 octobre 1941 - 10 novembre 1995), opposant au régime nigérian de l'époque, se trouvait alors incarcéré. II fut plus tard exécuté. 
de " docu-fictions " (Jedlowski, 2012 : 244) en raison du traitement de leurs sources, sont produits et tournés en diaspora avec de gros budgets; ils visent des audiences de salles de cinéma et reflètent la vie diasporique (Krings et Okome, 2013a : 7). Man on Ground met en images les relations tendues entre immigrants nigérians et leurs hôtes sud-africains, un sujet que le réalisateur du film connaît bien pour l'avoir vécu lui-même. En 1991, son père Kole Omotoso, universitaire et écrivain nigérian de renom, s'était installé en Afrique du Sud avec sa famille, ce qui fait d'Akin un Sud-Africain autant qu'un Nigérian. Dans un entretien, Fabian Lojede, I'un des principaux acteurs, confirme que " l'idée du film est née de [...] l'image d'Ernesto Nhamuavhe, le Mozambicain brûlé vif au cours des émeutes xénophobes de 2008. Et la première fois que j'ai vu cette photo, j'ai été profondément choqué " (Devriendt, 2013). Un forum en ligne donne plus de détails à ce sujet : "Le film s'est inspiré des événements qui se sont déroulés dans la soirée du dimanche 11 mai 2008, où un gang de jeunes gens de la cité noire d'Alexandra à Johannesburg se sont introduits dans un foyer de la rue de Londres et ont violemment attaqué ceux des résidents qu'ils considéraient comme étrangers. Ça a été l'étincelle qui a mis le feu aux poudres, déchaînant la molestation et le meurtre des étrangers et le pillage de leurs biens. Cette violence s'est ensuite propagée en quelques jours d'Alexandra aux agglomérations sauvages de Diepsloot et d'East Rand, où un Mozambicain, Ernesto Nhamuavhe, a été brûlé vif sous les rires des badauds. Connu dans le monde entier sous le nom de I'“'homme en feu", Ernesto Nhamuavhe, marié et père de famille, est à la source du film Un homme à terre ${ }^{13}$. La destruction est au cœur de ce film où tout est symbole, représentée par le symbole de l'allumette que I'on craque et du feu qui se propage, détruisant cabanes en tôle, symbole de la condition précaire des travailleurs noirs, et voitures, symbole des biens de consommation auxquels ces gens ne peuvent pas accéder.

Nollywood bénéficie depuis quelque dix ans d'une popularité croissante dans toute l'Afrique australe (Becker, 2013 : 180). Man on Ground semble cependant, au premier abord, très différent de ces films, aussi bien par son sujet que par son traitement des données. Ce qui frappe d'abord dans le film, c'est sa sobriété. Le décor est quasi inexistant, réduit à l'essentiel : une rue déserte, un terrain vague, une cage d'escalier, une pièce vide où ne se trouvent que deux chaises et une table. L'absence de couleurs elle-même, contrastant avec les costumes et les pagnes chatoyants habituels de Nollywood, participe de la sobriété de ce sombre récit dont la moitié se déroule de nuit dans des paysages lunaires baignés dans un brouillard opaque, des paysages industriels de rails, de machines et de couloirs interminables et nus qui amplifient les sentiments des personnages. L'absence totale de marqueurs spatiaux spécifiques donne en outre au film une couleur neutre permettant à tous, quelle que soit leur nationalité, de se retrouver dans ce récit qui pourrait se dérouler n'importe où - l'important n'est pas l'endroit, mais ce qui s'y passe et qui marque ceux qui vivent ces événements.

Le film, bâti sur les gros plans qui mettent l'accent sur les visages, les physionomies, pénètre d'emblée à l'intérieur des personnages, un choix souligné par l'usage du ralenti caractérisant un certain nombre de scènes, des retours en

13 Cf. http://www.nairaland.com/745001/nigerian-directed-film-man-ground 
arrière et des voix hors champ qui extériorisent les pensées et relient présent et passé. II est aussi I'histoire d'individus isolés : Ade vit une expérience solitaire de recherche de son frère ; la fiancée de Femi, seule et enceinte, est désespérée de ne pas savoir où il se trouve; le directeur de la fabrique est profondément seul avec ses secrets et détesté de ses ouvriers, et ne sait même pas que l'un d'entre eux a tué Femi ; sa femme elle-même reste murée dans le silence. II n'y a ici pour les migrants, ni couple, ni famille, ni ami, ni soutien - la mère de Femi et d'Ade, bien loin de là dans son village du Nigeria, est seulement évoquée, représentée par le petit paquet destiné à Femi et qui ne sera ouvert qu'à la fin par la jeune veuve. La vie de ces personnages sans racines, en transit et profondément étrangers à leur environnement, est perpétuellement menacée. Plongés au cœur d'une violence silencieuse au sein même de la communauté noire en lutte pour I'amélioration de ses conditions de vie, ils restent étrangers à cette communauté dont ils ne partagent ni la langue, ni la culture : la communication entre Ade et le patron de Femi est réduite au minimum - ils n'échangent que des monosyllabes. Le silence est au cœur du film où seuls les visages crient les sentiments : la peur, l'angoisse, le doute, le mépris, l'amour, disant l'impossibilité de communiquer, le manque de mots et de confiance en l'autre. Un bel exemple de ce silence est la soirée qu'Ade passe à boire enfermé en compagnie du patron de Femi et au cours de laquelle les deux hommes se saoulent en silence à la même bouteille, espérant peut-être arriver ainsi à briser le mur qui les sépare.

Le scénario tente de démêler un écheveau d'informations disparates, de retrouver les maillons manquants de la chaîne qui relie les personnages. Aucun de ceux-ci ne communique avec l'autre autrement que par des questions qui restent sans vraie réponse : où vit Femi ? Où travaille-t-il ? Pourquoi a-t-il choisi de travailler dans ce pays et dans ce secteur chaud? Qu'est-il devenu ? Pourquoi a-t-il été tué ? Ade est-il lui-même en danger ? Le mot " étranger " est prononcé plusieurs fois dans le film, soulignant l'impossible intégration du Nigérian Femi au sein de la communauté noire auprès de laquelle il vivait. L'Afrique du Sud de l'après-apartheid est présentée ici comme un pays africain, mais viscéralement hostile aux étrangers, accusés de mépriser les locaux et de leur prendre le peu qui leur revient. Le style comme la mise en scène de ce film rendent bien le profond isolement des immigrants et leur situation - coupés de leurs racines, sans nouvelles de leurs familles, sans soutien et sans grand espoir. Alors même qu'Ade a réussi à se faire une place en Angleterre, l'avenir de Femi reste un rêve irréalisé, sa vie est un échec à peine compensé par la promesse de l'enfant à naître. C'est là une sombre réflexion sur l'immigration sud-sud.

\section{Un outil pédagogique}

Dans la première partie de l'ouvrage issu de sa thèse, Dovey, elle-même sud-africaine, se penche sur la production cinématographique de son pays et suggère que le film reste le meilleur moyen d'atteindre les $75 \%$ de la population restés illettrés. Elle considère les difficultés des cinéastes sud-africains aux prises avec un passé de brutalité et de violence blanches et leur désir d'œuvrer à la réconciliation et à l'unité, et fait de son livre une critique de la xénophobie et un plaidoyer en faveur du multiculturalisme (Dovey, 2009 : 143). Cette réflexion est à l'arrière-plan de celle exprimée par le réalisateur de Man on Ground qui, comme d'autres cinéastes, " a choisi le réalisme pour représenter la violence, à 
des fins éducatives " (Dovey, $2009: 32$ ), dénonçant " les abus, le rejet, la peur et l'intolérance, suscitant la réflexion du public et son ouverture à plus de sagesse et d'humanité " (Crouillère, $2010: 190)$.

Interrogé sur le travail de production qui a précédé la sortie du film, Omotoso évoque d'abord brièvement les émeutes xénophobes qui ont déchiré l'Afrique du Sud en mai 2008 et qui ont servi de base au scénario, ajoutant qu' « il y avait eu des tensions auparavant, mais que c'était la première fois que se produisait une violente attaque concentrée [en un lieu] ". Pour lui, ce film ne fait que poursuivre le dialogue entre arts et société lancé à la suite de ces émeutes, lesquelles ont engendré de multiples réponses artistiques de soutien aux victimes. Le film est le résultat de trois mois de recherches approfondies entreprises par l'équipe de production sous la direction du réalisateur et des acteurs principaux, Fabian Lojede et Hakeem Kae-Kazim, auxquels s'est jointe la productrice Rosie Motene au début de 2011. Le script est né de l'analyse des émeutes, soutenue par la lecture d'ouvrages, d'entretiens et de commentaires sur la situation. Pour Omotoso, " il était important d'obtenir tous les points de vue sur I'histoire, et la recherche était cruciale. Ce travail nous a donné une idée de ce qui se passait sur le terrain et nous a fourni notre devise : "dites-leur que nous sommes d'ici". L'une des victimes, à qui l'on demandait ce qu'il dirait à ses assaillants s'il pouvait leur parler, répondit, "dites-leur que nous sommes d'ici". Ici, c'est-à-dire sur la planète terre. [...] La rédaction du script a pris trois ans. Nous avons d'abord poursuivi notre recherche, considéré les diverses options narratives, et fini par choisir la version définitive ${ }^{14}$.

Une enquête d'opinion menée en 2008 par l'institut World Values Survey a révélé que " l'Afrique du Sud arrive en tête des pays les plus xénophobes de la planète "(Pitron, 2012b). Vingt ans après la fin de l'apartheid, les discours introspectifs et les critiques émanant du cinéma sud-africain contemporain, s'ils ont sans nul doute été inspirés par les délibérations de la Commission Vérité et Réconciliation, sont aujourd'hui le reflet d'autres formes de violence, en particulier de la haine et du racisme envers les Noirs des dernières années (Dovey, 2009 : 53-54). Comme le notait Crouillère (2010 : 175), "le thème de l'immigration dans le cinéma africain a beaucoup changé au fil des années. La perception qu'on en a, également ". Né en réaction à l'explosion xénophobe de 2008, Man on Ground, film de grande qualité technique et cinématographique produit en partenariat avec la Fondation sud-africaine du film et de la vidéo et I'Organisation internationale pour les migrations (OIM) après une campagne de fonds ouverte à la population tout entière, est considéré comme " une exploration très actuelle sur les difficultés rencontrées par les réfugiés ${ }^{15}$. Sa spécificité est inscrite dans I'histoire de sa production : de la conception à la projection, il s'agit là d'un film engagé, au confluent des expériences personnelles du réalisateur et de l'histoire récente du pays. Il se distingue en outre en ce qu'il a été choisi comme outil de réconciliation, en raison de son histoire et de ses qualités : chacune de ses projections est une étape de la campagne engagée à travers tout le pays et au-delà contre la xénophobie qui accompagne si souvent la pauvreté.

14 Cf. http://www.spling.co.za/movie-news/interview-akin-omotoso-on-his-film-man-onground

15 Omotoso Akin (2012) Man on Ground: We are from here Campaign, 30 avril, [en ligne] consulté le 30/06/2016. URL : https://www.facebook.com/ManOnGround 
Lancé au festival du film de Toronto au Canada en 2011, Man on Ground parcourt aujourd'hui l'Afrique du Sud : des projections du film se sont déroulées depuis dans quatre provinces sud-africaines, à Musina, Malelane, Durban et Cape Town à l'occasion de la campagne d'information "Dites-leur que nous sommes d'ici ". Cette campagne cherche à informer la population sur les difficultés rencontrées par les immigrants, à faciliter le dialogue entre migrants et communautés locales et à œuvrer en faveur de la diversité, de la tolérance et de la paix ${ }^{16}$. Soutenue par l'Organisation internationale pour les migrations (OIM), le Goethe Institut et I'Open Society Initiative of Southern Africa (OSISA) et dirigée par le réalisateur du film, elle suit une autre campagne précédemment organisée par l'OIM et intitulée "Moi aussi je suis un migrant ", qui cherchait à promouvoir la paix en aidant les diverses communautés sud-africaines à réaliser que l'expérience de la migration concerne tous les gens, tôt ou tard, et devait être célébrée. Le soutien apporté à la diffusion du film par I'OIM est dans la droite ligne de la stratégie de cette institution, qui vise à informer sur la situation des migrants, favoriser la compréhension des questions de migration, promouvoir la protection des travailleurs migrants et de leur famille, et à promouvoir, faciliter et appuyer le débat et le dialogue sur la migration ${ }^{17}$. Ce soutien consacre la reconnaissance de l'adéquation entre la mission du film d'Omotoso et celle de I'OIM, et démontre à l'évidence à quel point l'outil cinématographique peut contribuer à l'éducation populaire. Les projections du film sont suivies d'entretiens filmés visant à donner aux spectateurs l'occasion de partager leur expérience et leur opinion concernant les notions de xénophobie, d'identité, d'intégration, de cohésion sociale et d'exclusion (Dixon, 2013).

\section{Conclusion}

L'importance de ce film, icône de l'expérience nigériane contemporaine sur la migration sud-sud et l'altérité, le place à la charnière entre deux mondes. Noirs, Africains et pourtant étrangers en Afrique du Sud, les Nigérians représentent ici tout un continent à la recherche de la stabilité, à travers un film qui sert de creuset à une réflexion sur la difficulté de l'intégration hors de chez soi. Le nombre de sélections officielles de Man on Ground à Toronto, Lagos et Dubaï en 2011, à Berlin et Durban en 2012, et les prix reçus depuis ${ }^{18}$, prouvent amplement le retentissement du film et de son message. Ce sont cependant les gens ordinaires et les communautés que cherche surtout à toucher Man on Ground, comme I'explique son réalisateur : "Je voulais un film qui stimule le dialogue aussi bien au sein des communautés affectées par la violence xénophobique qu'à travers le monde. [...] Nous avons encouragé les gens à s'impliquer dans notre campagne Facebook "Man on Ground : dites-leur que nous sommes d'ici". J'espère que ce film va changer la mentalité des gens qui s'obstinent à persécuter leurs pareils soit parce qu'ils ne "nous ressemblent pas" ou qu'ils "ne parlent pas comme nous" $"{ }^{19}$.

16 Ibid.

17 Cf. http://www.iom.int/fr/enonce-de-mission

18 Meilleur film au festival du film de Johannesburg ; meilleure équipe d'acteurs au festival du film de charité (Monaco, 2012) ; meilleur film sud-africain au 10e festival du film tricontinental (2012); meilleur film pour son réalisateur, sa cinématographie, son édition et sa qualité de son, Africa Magic, (Lagos, 2013), etc.

19 Cf. http://www.flavourmag.co.uk/review-man-on-ground/ 
Ces propos confirment l'appartenance du film d'Omotoso à Nollywood, cinéma populaire et didactique. Man on Ground, réalisé par un immigrant nigérian en Afrique du Sud, et si éloigné des productions nollywoodiennes traditionnelles, de leurs foules, de leurs discours et de leur exubérance, s'y rattache cependant par sa critique des maux observés et par sa volonté d'enseignement et de changement social. Dans un contexte africain marqué par la difficulté de circulation des livres et le manque de bibliothèques, il fait partie de ces productions " devenues un moyen de communiquer l'expérience diasporique et les difficultés qu'elle comporte à tous ceux qui sont restés au pays " (Krings et Okome, 2013a : 7). Production multilingue, au confluent de diverses cultures et mêlant l'anglais, le zulu, le sotho et le yoruba, il s'inscrit également au nombre des films porteurs d'un message de paix au service de la campagne actuelle contre la violence en Afrique du Sud et ailleurs.

\section{Références bibliographiques}

Agber Kwaghkondo (2014) Thematic developments in Nigerian video films, in Foluke Ogunleye Ed., African Film: Looking back and Looking Forward, Cambridge, Cambridge Scholars Publishing, pp. 222-231.

Aina Olatunji Francis and Olorunshola Derin Augustine (2008) Alcohol and substance use portrayals in Nigerian video tapes: An analysis of 479 films and implications for public drug education, International Quarterly of Community Health Education, 28 (1), pp. 63-71.

Barrot Pierre (2005) Nollywood : le phénomène vidéo au Nigeria, Paris, L'Harmattan, $175 \mathrm{p}$.

Becker Heike (2013) Nollywood in urban Southern Africa: Nigerian video films and their audiences in Cape Town and Windhoek, in Matthias Krings and Onookome Okome Eds., Global Nollywood: The transnational dimensions of an African video film industry, Bloomington, $\mathrm{OH}$, Indiana University Press, pp. 179-198.

Botha John (2014) South-African film: Looking back and looking forward, in Foluke Ogunleye Ed., African Film: Looking back and Looking Forward, Cambridge, Cambridge Scholars Publishing, pp. 20-34.

Crouillère Monique (2010) Cinéma africain et immigration, in Jean Ouedraogo Éd., Figuration et mémoire dans les cinémas africains, Paris, L'Harmattan, pp. $175-192$.

Deveny Thomas G. (2012) Migration in Contemporary Hispanic Cinema, Lanham, Scarecrow Press, $406 \mathrm{p}$.

Dovey Lindiwe (2009) African Films and Literature. Adapting Violence to the Screen, New York, Columbia University Press, 334 p.

Haynes Jonathan (2013) The Nollywood diaspora: A Nigerian video genre, in Matthias Krings and Onookome Okome Eds., Global Nollywood: The transnational dimensions of an African video film industry, Bloomington, $\mathrm{OH}$, Indiana University Press, pp. 73-99.

Hungbo Jendele (2014) Migrating Nollywood: Melting borders in Tunde Kelani's Abeni, in Foluke Ogunleye Ed., African Film: Looking back and Looking Forward, Cambridge, Cambridge Scholars Publishing, pp. 171-182. 
Ibeanu Okechukwu and Orji Nkwachukwu (2004) Despotism, Democracy and Diplomacy: Nigeria - South Africa Relations 1993-1999, Democracy \& Development: Journal of West African Affairs, 4 (2), pp. 49-66.

Jedlowski Alessandro (2015) Nigerian migrants, Nollywood videos and the emergence of an anti-humanitarian representation of migration in Italian cinema, in Emma Bond, Guido Bonsaver and Federico Faloppa Eds., Destination Italy: Representing Migration in Contemporary Media and Narrative, Oxford, Peter Lang, pp. 397-414.

Jedlowski Alessandro (2013) From Nollywood to Nollyworld: Processes of transnationalization in the Nigerian video film industry, in Matthias Krings and Onookome Okome Eds., Global Nollywood: The transnational dimensions of an African video film industry, Bloomington, $\mathrm{OH}$, Indiana University Press, pp. 25-45.

Jedlowski Alessandro (2012) On the periphery of Nollywood: Nigerian video filmmaking in Italy and the emergence of an intercultural aesthetics, in Cristina Lombardi-Diop and Caterina Romeo Eds., Postcolonial Italy: Challenging National Homogeneity, New York, Palgrave McMillan, pp. 239-252.

Krings Matthias and Okome Onookome (2013a) Nollywood and its diaspora: An introduction, in Matthias Krings and Onookome Okome Eds., Global Nollywood: The transnational dimensions of an African video film industry, Bloomington, $\mathrm{OH}$, Indiana University Press, pp. 1-22.

Krings Matthias and Okome Onookome (Eds.) (2013b) Global Nollywood: The transnational dimensions of an African video film industry, Bloomington, $\mathrm{OH}$, Indiana University Press, $371 \mathrm{p}$.

Ogunleye Foluke (Ed.) (2014) African Film: Looking back and Looking Forward, Cambridge, Cambridge Scholars Publishing, 265 p.

Osoba Olusegun and Fajana Adewunmi (1999 [1980]) Educational and social development during the twentieth century, in Obaro lkime Ed., Groundwork of Nigerian History, Ibadan, Heinemann, pp. 570-600.

Ouedraogo Jean (Éd.) (2010) Figuration et mémoire dans les cinémas africains, Paris, L'Harmattan, $258 \mathrm{p}$.

Pype Katrien (2013) Religion, migration and media aesthetics, in Matthias Krings and Onookome Okome Eds., Global Nollywood: The transnational dimensions of an African video film industry, Bloomington, $\mathrm{OH}$, Indiana University Press, pp. 199-222.

Reich Courseuil Anelise (2006) Film beyond boundaries: Film, migrant narratives and other media, Ilha de Desterro, Journal of English language, literature in English and Cultural Studies, 51, pp. 9-18.

Rofheart Mahriana (2014) Shifting Perceptions of Migration in Senegalese Literature, Film and Social Media, Plymouth, Lexington Books, 157 p.

Schrader Sabine and Winkler Daniel (Eds.) (2013) The Cinemas of Italian Migration: European and Transatlantic Narratives, Cambridge, Cambridge Scholars publishing, $340 \mathrm{p}$.

Tati Gabriel (2008) The immigration Issues in the Post-Apartheid South Africa: Discourses, Policies and Social Repercussions, Espace populations sociétés, 3, pp. 423-440, [en ligne] consulté le 26/08/2015. URL : http://eps.revues.org/3496 
Tshibambe Germain Ngoie (2011) Karachika! Nigerian Movies and Nigerian Migrants in the "Glocal City". The Case of Lubumbashi, Paper presented to the Panel on "Migration and African Cities: International Dimensions" at the $4^{\text {th }}$ European Conference on African Studies, 15-18 June, Uppsala, Sweden.

Tsika Noah A. (2015) Nollywood Stars, Media and Migration in West Africa and the Diaspora, Bloomington, Indiana University press, $384 \mathrm{p}$.

Ugochukwu Françoise (2013) Nollywood on the Move, Nigeria on Display, Trier, Wissenschaftlicher Verlag, $260 \mathrm{p}$.

Ugochukwu Francoise (2012) Nollywood on the road - Nigeria on display, in Sunday Enessi Ododo Ed., Fireworks for a Lighting Aesthetician: Essays and Tributes in Honor of Duro Oni @ 60, Lagos, Centre for Black and African Arts \& Civilization (CBAAC), pp. 465-475.

Véron Jean-Bernard (2006) L'Afrique du Sud et le Nigeria : du maintien de la paix à la recherche d'un positionnement stratégique sur le continent africain, Afrique contemporaine, $219(3)$, pp. 163-172.

Weiss Thomas Lothar (1998) Migrants nigérians : la diaspora dans le sud-ouest du Cameroun, Paris, L'Harmattan, 272 p.

\section{Sites Internet}

20 minutes (2013) L'Afrique du Sud reçoit le président du Nigeria pour relancer la coopération, 20 minutes, 7 mai, [en ligne] consulté le 30/06/2016. URL : http:// www.20minutes.fr/monde/1151803-20130507-20130507-afrique-sud-recoit-president-nigeria-relancer-cooperation

BellaNaija.com (2015) Xenophobic Attacks in South Africa: Nigerians Lose over N21 Million, Bella Naija, April 20, [online] last checked on 30/06/2016. URL: http:// www.bellanaija.com/2015/04/20/xenophobic-attacks-in-south-africa-nigerianslose-over-n21-million/

Brock Joe and Dludla Nqobile (2015) Xenophobia row exposes rivalry between South Africa and Nigeria, Reuters, April 28, [online] last checked on 30/06/2016. URL: http://www.reuters.com/article/2015/04/28/us-safrica-nigeria-tensionsidUSKBNONJ1OL20150428

Cessou Sabine (2015) D'Afrique vers I'Europe : " Hope ", un film rude sur les migrants (entrevue avec Boris Lojkine), L'Obs avec Rue 89, 29 janvier, [en ligne] consulté le 30/06/2016. URL : http://blogs.rue89.nouvelobs.com/ruesdafriques/2015/01/29/du-maroc-leurope-hope-un-film-rude-sur-les-migrantsafricains-234161

Devriendt Tom (2013) Making "Man on Ground", Africa is a country, April 10, [online] last checked on 30/06/2016. URL: http://africasacountry.com/2013/04/10/ making-man-on-ground

Dixon Gaone (2013) Man on Ground, un film contre la xénophobie projeté en Afrique du Sud, Organisation Internationale pour les Migrations (OIM), 4 mai, [en ligne] consulté le 30/06/2016. URL : http://www.iom.int/fr/news/man-groundun-film-contre-la-xenophobie-projete-en-afrique-du-sud

Fisher Jonah (2011) Nigeria's thriving economy lures expats back home, $B B C$ News, October 20, [online] last checked on 30/06/2016. URL: http://www.bbc. co.uk/news/world-africa-15382981 
Mboungou Vitraulle (2012) L'immigration africaine en Afrique : les migrants " boucs émissaires ", Afrique-Expansion magazine, 13 septembre, [en ligne] consulté le 30/06/2016. URL : http://geram862.mywhc.ca/archives/limmigration-africaine-en-afrique/5131-limmigration-africaine-en-afrique-les-migrants-Iboucs-emissaires-r.html

Naij.com (2013) Why do South Africans hate Nigerians?, Naij.com, [online] last checked on 30/06/2016. URL: https://www.naij.com/37699.html

Nséké Léopold (2012) L'immigration africaine en Afrique : les pays d'accueil, Afrique-Expansion magazine, 10 septembre, [en ligne] consulté le 30/06/2016. URL : http://geram862.mywhc.ca/archives/limmigration-africaine-enafrique/5106-limmigration-africaine-en-afrique-les-pays-daccueil.html

Pitron Guillaume (2012a) African odysseys turn to the south, Le Monde diplomatique, 14 novembre, [online] last checked on 30/06/2016. URL: http://mondediplo. com/2012/11/14african-immigration

Pitron Guillaume (2012b) Avec les migrants africains en route pour Johannesburg, Le Monde diplomatique, octobre, [en ligne] consulté le 30/06/2016. URL : http:// www.monde-diplomatique.fr/2012/10/PITRON/48239

Premium Times (2015) FACT-CHECK: Xenophobia: Immigrants to South Africa not stealing jobs as claimed, Premium Times, April 17, [online] last checked on 30/06/2016. URL: http://www.premiumtimesng.com/news/headlines/181605-factcheck-xenophobia-immigrants-to-south-africa-not-stealing-jobs-as-claimed.html

\section{Filmographie ${ }^{20}$}

Anyaene Chineze (2010) lje the Journey, Jos et Los Angeles (metteurs en scène : Chineze Anyaene et Paula Moreno ; acteurs : Omotola Ajalade-Ekeinde, Genevieve Nnaji, Ulrich Que, Jeff Swarthout, Clem Ohameze, Jon Morgan Woodward et John Ammons; langues : anglais et igbo).

Chidebe Mac Collins (2006) Brainwash (metteur en scène : Chijioke Nneji ; acteurs : John Okafor, Charles Awurum, Kinsley Orji, Uche Elendu et Jim Lawson ; langues : anglais et igbo).

Chine Vigil (2006) African Dilemma.

Ejiro Chico (2004) Life in New York (metteur en scène : Chico Ejiro ; acteurs : Desmond Elliot, Jim lyke, Pascal Âtmâ, Fadekemi Balogun, Paul Obazele et Veronica Kelly).

Fana Austin (2012) The King's Secret (metteur en scène : Peter Okonkwo ; acteurs: Mike Ezuruonye, Jibola Dabo et Hacha Eke).

Lojkine Boris (2014) Hope (acteurs : Justin Wang, Endurance Newton et Nabyl Fally Koivogui).

Louwrens Paul (2005) Coming to South Africa (metteur en scène : Hakeem Kae-Kazim ; acteurs : Jade Hands, Hakeem Kae-Kazim et Ramsey Nouah).

Moses Gabriel (2002) One Dollar (metteurs en scène : Damian et Gabriel Moses ; acteurs : Patience Ozokwor, Victor Osuagwu, Bob-Manuel Udokwu, Chiege Alisigwe et Jim lyke).

20 Dans certains cas, il n'a pas été possible de trouver tous les détails concernant les films, du fait que beaucoup d'entre eux ne sont pas accessibles. Le lieu du tournage n'a été mentionné que lorsqu'il a une importance particulière. 
Njamah Aquila et Ogoro Kinsley (2007) Widow (metteurs en scène : Agatha Nwakalor et Eloho Ovie-Ogoro ; acteurs : Stella Damasus, Yemi Solade et Peter Bunor).

Odife lyke (2011) The American villager (metteur en scène : Dubem Chigbo Onyemesili ; acteurs : John Okafor, Patience Ozokwor, Chinwetalu Agu, Leo Mezie et Charity Eke).

Okoro Kingsley (2003) Osuofia in London (metteur en scène : Kingsley Okoro ; acteurs : Nkem Owoh, Mara Dewent et Charles Angiama).

Omoigui Vincent et Sandretti Simone (2009) Akpegi Booz, Turin.

Omotoso Akin (2003) God is African (acteurs : Hakeem Kae-Kazim, Sami Sabiti, Esmeralda Bihl et Hugh Masebenza).

Omotoso Akin, Kae-Kazim Kazeem, Motene Rosie et Adeoye Fabian (2011) Man on Ground (metteur en scène : Akin Omotoso ; acteurs : Hakeem Kae-Kazim, Fabian Adeoye, Fana Mokoena et Buku Mazibuko ; langues : anglais, zulu, sotho et yoruba).

Opeoluwa Simi (2004) Fateful Love (metteur en scène : Andy Best Nnadi ; acteurs : Uche Ama Abriel, Omotola Jalade-Ekeinde, Ramsey Noah, Patience Ozokwor, Paul Obazele, Sam Obiakeme et Trine Thieelen).

Williams Adim (2004) Mr. Ibu in London (metteur en scène : Chukwuka Emelionwu ; acteurs : John Okafor, Kareem Adepoju et Marian Davies). 


\section{Françoise Ugochukwu}

\section{$\cdots$ Migrations nigérianes à l'écran : Man on Ground et l'Afrique du Sud post-apartheid}

La réflexion sur la migration est depuis longtemps l'un des grands thèmes de la littérature africaine. Qu'en est-il du cinéma ? Un grand nombre de films sur les itinéraires et l'expérience des migrants d'autres continents ont déjà été produits en Occident, mais rares sont les films africains sortis sur le sujet. Au moment même où la montée du malaise diplomatique entre le Nigeria et l'Afrique du Sud se trouve largement répercutée dans les médias, cet article considère l'évocation de l'étranger dans la production nollywoodienne et l'évolution de son traitement de la migration depuis les débuts. II brosse un tableau des difficultés rencontrées par les migrants africains en Afrique du Sud, pour mieux contextualiser le film Man on Ground (2011) du Sud-Africain d'origine nigériane Akin Omotoso, dont I'analyse est I'objet de I'article.

\section{Nigerian Migration on Screen: Man on Ground and Post-Apartheid South Africa}

The reflection on migration has been one of the main themes of African literature for some time. What about cinema? Many Western films have already been produced on migrants from other continents, their itineraries and experience, yet African films on the subject are few. This article considers the presentation of foreign lands in Nollywood films and the evolution of migration stories since the 1990s, in the light of the growing diplomatic malaise affecting bilateral relations between Nigeria and South Africa, widely discussed in the media. It equally reports on recent problems encountered by Nigerian migrants in South Africa as a background to the film Man on Ground (2011) produced and directed by the Nigeria-born South African Akin Omotoso, whose study is the subject of the article.

\section{Migraciones nigerianas en pantalla: Man on Ground y Sudáfrica post-apartheid}

La reflexión sobre la migración es desde hace tiempo uno de los grandes temas de la literatura africana. ¿Y qué ocurre con el cine? Un buen número de películas sobre los itinerarios y la experiencia de los migrantes de otros continentes ya han sido producidas en Occidente, pero las películas africanas sobre el mismo tema son más escasas. En el momento en que la tensión diplomática entre Sudáfrica y Nigeria tiene una alta repercusión en los medios de comunicación, este artículo aborda la consideración del extranjero en la producción nollywodiense y la evolución del tratamiento de la migración desde sus orígenes. El texto plantea las dificultades que encuentran los migrantes africanos en Sudáfrica, con el fin de contextualizar la película Man on Ground (2011) del sudafricano de origen nigeriano Akin Motoso, cuyo análisis es el objeto del artículo. 